\title{
Superforms, supercurrents, minimal manifolds and Riemannian geometry
}

\section{Bo Berndtsson ${ }^{1}$}

Received: 16 June 2019 / Revised: 9 October 2019 / Accepted: 19 December 2019 /

Published online: 14 January 2020

(c) The Author(s) 2020

\begin{abstract}
Supercurrents, as introduced by Lagerberg, were mainly motivated as a way to study tropical varieties. Here we will associate a supercurrent to any smooth submanifold of $\mathbb{R}^{n}$. Positive supercurrents resemble positive currents in complex analysis, but depend on a choice of scalar product on $\mathbb{R}^{n}$ and reflect the induced Riemannian structure on the submanifold. In this way we can use techniques from complex analysis to study real submanifolds. We illustrate the idea by giving area estimates of minimal manifolds and a monotonicity property of the mean curvature flow. We also use the formalism to give a relatively short proof of Weyl's tube formula.
\end{abstract}

\section{Introduction}

A superform on $\mathbb{R}^{n}$ is defined as a differential form on $\mathbb{C}^{n}$ whose coefficients do not depend on the imaginary part of the variable. The dual of the space of superforms (with coefficients compactly supported in $\mathbb{R}^{n}$ and with the usual topology from the theory of distributions), is the space of supercurrents. Superforms and supercurrents were introduced by Lagerberg (2012), as a way to study tropical varieties. A tropical variety in $\mathbb{R}^{n}$ defines a $d$-closed, positive, supercurrent of integration, and conversely any such supercurrent defines a tropical variety, given a condition on the dimension of the support. (See also the work of Babaee (2014), for related work using standard currents on $\left(\mathbb{C}^{*}\right)^{n}$ instead of supercurrents.)

Here we extend the 'superformalism' in a different direction by associating to any smooth (or piecewise smooth) submanifold, $M$, of $\mathbb{R}^{n}$ a supercurrent, $[M]_{s}$, with the aim to apply methods from complex analysis to real manifolds. These supercurrents are $d$-closed only if the manifold is a linear subspace, but $d[M]_{S}$ is given by an explicit formula involving the second fundamental form of $M$. As a result, it turns out that $M$ is minimal if and only if

\footnotetext{
$凶$ Bo Berndtsson

bob@chalmers.se

1 Department of Mathematics, Chalmers University of Technology, 41296 Göteborg, Sweden
} 


$$
[M]_{s} \wedge \beta^{m-1} /(m-1) !
$$

is closed, where $m$ is the dimension of $M$ and $\beta$ is the Euclidean Kähler form on $\mathbb{C}^{n}$ (Corollary 5.2). Thus, minimality is characterized by a rather simple linear equation, which suggests a generalization of minimal manifolds to minimal 'supercurrents'. This is of course similar to the use of (classical) currents and varifolds in the theory of minimal manifolds, but has the extra feature of a bidegree, as in complex analysis. With this we can imitate Lelong's method for positive closed currents to prove e. g. the monotonicity formula for minimal manifolds, and a volume estimate that generalizes a recent result of Brendle and Hung (2017) (Theorem 6.3). We also obtain a result on removable singularities for minimal manifolds along the lines of the El Mir-Skoda theorem from complex analysis (Theorem 7.2), and a formula for the variation of the volume under the mean curvature flow (Theorem 8.2). ( I take the opportunity to thank Duong Phong for suggesting to apply the formalism to the mean curvature flow.)

After that we give an expression of the Riemann curvature tensor of $M$ as a superform. This is basically a rewrite of Gauß's formula. We apply it in the last section, to give a rather short proof of Weyl's tube theorem, Weyl (1939). The proof is in essence the same as Weyl's proof, but we have included it, hoping to show that the superformalism is useful in computations.

Finally I would like to thank an anonymous referee for a very careful reading of my manuscript, spotting many errors and suggesting several improvements.

\section{Preliminaries}

We start by recalling the definitions and basic properties of superforms and supercurrents, mainly following Lagerberg (2012) (and Berndtsson (2006)), but with some modifications. Let $E$ be an $n$-dimensional vector space over $\mathbb{R}$. Thus $E$ can be identified with $\mathbb{R}^{n}$, but at some points it will be convenient not to fix a basis. We define the 'superspace' of $E$ to be

$$
E_{s}=E \oplus E=E_{0} \oplus E_{1}
$$

where we use the subscripts to indicate the first or second summand. A superform on $E$ is a differential form on $E_{S}$ that is invariant under translation in the $E_{1}$-variable. If $x=\left(x_{1}, \ldots x_{n}\right)$ and $\xi=\left(\xi_{1}, \ldots \xi_{n}\right)$ are coordinates on $E_{0}$ and $E_{1}$ respectively a superform can then be written

$$
a=\sum a_{I, J}(x) d x_{I} \wedge d \xi_{J}
$$

where the coefficients do not depend on $\xi$. We say that a superform $a$ has bidegree $(p, q)$ if the lengths of the multiindices in (2.1) satisfy $|I|=p$ and $|J|=q$. (Notice that this is not the same bigrading as in the complex case.) With these conventions, a superform of bidegree $(0,0)$ can be identified with a function on $E$, since it does not depend on $\xi$. So, a 'superfunction' on $E_{S}$ is a function on $E$. 
We also equip $T^{*}\left(E_{S}\right)=T^{*}\left(E_{0}\right) \oplus T^{*}\left(E_{1}\right)$ with a complex structure $J$, such that $J$ maps $T^{*}\left(E_{0}\right)$ to $T^{*}\left(E_{1}\right)$ and vice versa. (Here our definitions differ from Lagerberg's, who considers instead maps from $T^{*}\left(E_{0}\right)$ to $T^{*}\left(E_{1}\right)$ satisfying $J^{2}=1$.) In the sequel we will only consider bases of $E_{0}$ and $E_{1}$ such that $J\left(d x_{i}\right)=d \xi_{i}$, and therefore $J\left(d \xi_{i}\right)=-d x_{i}$. We then extend $J$ to act on forms of arbitrary bidegree so that $J(a \wedge b)=J(a) \wedge J(b)$. When $a$ is of bidegree $(p, 0)$ we sometimes write $J(a)=a^{\#}$.

The ordinary exterior derivative of $a$ is

$$
d a=\sum d a_{I, J} \wedge d x_{I} \wedge d \xi_{J}=\sum \frac{\partial a_{I, J}}{\partial x_{k}} d x_{k} \wedge d x_{I} \wedge d \xi_{J}
$$

and we also define

$$
d^{\#} a:=\sum d^{\#} a_{I, J} \wedge d x_{I} \wedge d \xi_{J}=\sum \frac{\partial a_{I, J}}{\partial x_{k}} d \xi_{k} \wedge d x_{I} \wedge d \xi_{J}
$$

$E_{s}$ is thus just the complexification of $E$ and $d^{\#}$ is just $d^{c}=i(\bar{\partial}-\partial)$, but we write $d^{\#}$ to emphasize that it acts only on superforms, i. e. forms not depending on $\xi$. Note that we also have

$$
d^{\#} a=(-1)^{k} J d J(a)
$$

where $k=p+q$ is the total degree of $a$.

We also suppose given a scalar product on $E$ and extend it to $E_{S}$ so that it is invariant under $J$ and $E_{0} \perp E_{1}$. Thus, if $d x_{i}$ are orthonormal, $d \xi_{i}=d x_{i}^{\#}$ are orthonormal on $E_{1}$ and $d x_{i}, d \xi_{i}$ are orthonormal on $E_{s}$.

The main point in the construction of $E_{s}$ is the definition of integrals. If $a$ is a form of maximal bidegree $(n, n)$, we write $a=a_{0} d x \wedge d \xi$ with $d x=d x_{1} \wedge \ldots d x_{n}, d \xi=d x^{\#}$ and put

$$
\int_{E_{s}} a=\int_{E_{0}} a_{0} d x \int_{E_{1}} d \xi
$$

The integral over $E_{0}$ here is well defined as soon as we have chosen an orientation of $E_{0}$, if we assume that $a_{0}$ has enough decrease at infinity to make the integral convergent. For the integral with respect to $\xi$ we define

$$
c_{n} \int d \xi=1
$$

if $d x_{i}$ are orthonormal and oriented, where $c_{n}=(-1)^{n(n-1) / 2}$. Except for the constant $c_{n}$, this is the Berezin integral (Berezin 1987). The reason for introducing the factor $c_{n}$ is that we want the integral of

$$
a=a_{0} d x_{1} \wedge d \xi_{1} \wedge \cdots d x_{n} \wedge d \xi_{n}
$$


to be positive if $a_{0}$ is positive in accordance with the complex case. If $a$ is given by (2.2) and $d x_{i}$ are orthonormal, we get

$$
\int_{\mathbb{R}_{s}^{n}} a=c_{n} \int_{\mathbb{R}_{s}^{n}} a_{0} d x \wedge d \xi=\int a_{0} d x c_{n} \int d \xi=\int a_{0} d \lambda(x),
$$

where $d \lambda$ is Lebesgue measure. Note that if we change orientation, the 'superintegral' remains the same. This follows since the integrals with respect to $x$ and $\xi$ both change sign, or directly from (2.3).

Let us briefly compare superintegration to classical integration over the complexification. The first problem with classical integration is of course that the classical integral over $E_{S}$ would always be divergent since $a_{0}$ does not depend on $\xi$. This could be overcome by replacing $E_{1}$ by its quotient by a lattice, so that we would replace $E_{s}$ by $E_{0} \times T^{n}$, where $T^{n}$ is a torus. The reason that does not work here is that we will later want to integrate forms of lower bidegree over linear subspaces, and these subspaces do not in general correspond to subtori, unless the subspace satisfies a rationality condition that we cannot assume to be satisfied.

We next list a few rules of computation for superintegrals. First

$$
\int_{\mathbb{R}_{s}^{n}} a=\int_{\mathbb{R}_{s}^{n}} J(a)
$$

since $J(a)=a$ if $a$ is of bidegree $(n, n)$. Furthermore, if $a$ is compactly supported of bidegree $(n-1, n)$,

$$
\int_{\mathbb{R}_{s}^{n}} d a=0
$$

This means that we have the usual formula for integration by parts

$$
\int_{\mathbb{R}_{s}^{n}} d a \wedge b=(-1)^{k+1} \int_{\mathbb{R}_{s}^{n}} a \wedge d b
$$

if at least one of the forms $a$ and $b$ is compactly supported. Similarly, using $d^{\#}= \pm J d J$ and that the superintegral is $J$-invariant, we also have

$$
\int_{\mathbb{R}_{s}^{n}} d^{\#} a=0
$$

This means that the same integration by parts formula as for $d$ also holds for $d^{\#}$. From this one verifies, for example, that if $\rho$ is a function and $S$ is of bidegree $(n-1, n-1)$, then

$$
\int_{\mathbb{R}_{s}^{n}} \rho d d^{\#} S=\int_{\mathbb{R}_{s}^{n}} d d^{\#} \rho \wedge S
$$


We can also define superintegrals over submanifolds of $\mathbb{R}^{n}$ if the form we integrate is of bidegree $(p, n)$, i. e. is of maximal degree in $d \xi$. For instance, if $D$ is a smoothly bounded domain in $\mathbb{R}^{n}$ and $a=\alpha \wedge d \xi$ is of bidegree $(n-1, n)$, then

$$
\int_{\partial D} a:=\int_{\partial D} \alpha \int_{E_{1}} d \xi=c_{n} \int_{\partial D} \alpha .
$$

It thus follows directly that Stokes' formula holds:

$$
\int_{\partial D} a=\int_{\mathbb{R}_{s}^{n}} \chi_{D} d a=: \int_{D_{s}} d a
$$

'Superintegration' over a submanifold of forms not of full degree in $d \xi$ is a different matter that will be discussed later in Sect. 4, but as a preparation for that we next discuss the case of linear subspaces. Let $F$ be a linear subspace of $E=E_{0}$ of dimension $m$. Then its complexification is $F_{s}=F \oplus J(F)$, so $F$ defines a superspace which is a complex linear subspace of $E_{s}$. Restricting a superform of bidegree $(m, m)$ on $E_{s}$ to $F_{S}$, we thus have a definition of the integral of $a$ over $F_{S}$,

$$
\int_{F_{s}} a
$$

as before.

Having defined superforms and their integrals, we now turn to supercurrents. The space of supercurrents of bidegree $(p, q)$, or bidimension $(n-p, n-q)$, is the dual of the space of smooth, compactly supported (in $x$ !), superforms of bidegree $(n-p, n-$ $q$ ). Here we use the classical notion of duals from the theory of distributions, and we say that a supercurrent is of order zero if it is continuous for the uniform topology on superforms. Note that a supercurrent of bidegree $(n, n)$ is a (classical) current of top degree on $E$, since superforms of bidegree $(0,0)$ are functions on $E$. In particular, a supercurrent of top degree and order zero is a measure on $E$ (not on $E_{S}$ ). As usual, given coordinates, a supercurrent can be written

$$
T=\sum T_{I, J} d x_{I} \wedge d \xi_{J}
$$

where $T_{I, J}$ are distributions on $E_{0}$. Let us explain this notation a bit more. We think of a distribution as having bidegree $(0,0)$ (a generalized function), i. e. as acting on forms of top degree. Then (2.5) means that if $a$ is a test form of complementary bidegree

$$
T . \alpha=\sum T_{I, J} \cdot\left(a_{K . L} d x_{I} \wedge d \xi_{J} \wedge d x_{K} \wedge d \xi_{L}\right)
$$

In particular, if $T_{I, J}$ are locally integrable functions, then

$$
T . \alpha=\int_{\mathbb{R}_{s}^{n}} T \wedge \alpha .
$$


In practice, for us, the coefficients will mostly be at worst measures. (The exception is Sect. 8 where we discuss the mean curvature flow.) If $\mu$ is a measure, it acts on functions, and so should be regarded as a current of bidegree $(n, n)$. We define the corresponding object of bidegree $(0,0), * \mu$, by

$$
* \mu . \alpha_{0} d x_{1} \wedge d \xi_{1} \wedge \cdots d x_{n} \wedge d \xi_{n}=\mu . \alpha_{0} .
$$

As an example of this we consider superintegration over a linear subspace $F$. Choose orthonormal coordinates so that

$$
F=\left\{x_{m+1}=\cdots x_{n}=0\right\}
$$

and write an $(m, m)$-form as

$$
a=a_{0} d x_{1} \wedge d \xi_{1} \cdots d x_{m} \wedge d \xi_{m}+\cdots
$$

Then the restriction of $a$ to $F_{s}$ is just $a_{0} d x_{1} \wedge d \xi_{1} \cdots d x_{m} \wedge d \xi_{m}$, and

$$
\int_{F_{s}} a=\int_{F} a_{0} d \lambda_{F}
$$

as before. Unwinding definitions, this means that the supercurrent defined by superintegration over $F$ is

$$
\begin{aligned}
{[F]_{s}: } & =\left(* d \lambda_{F}\right) d x_{m+1} \wedge d \xi_{m+1} \cdots d x_{n} \wedge d \xi_{m} \\
& =c_{n-m}\left(* d \lambda_{F}\right) d x_{m+1} \cdots \wedge d x_{n} \wedge d \xi_{m+1} \cdots \wedge d \xi_{n}
\end{aligned}
$$

Since the standard current of integration on $F$ as a subspace of $E$ is

$$
[F]=\left(* d \lambda_{F}\right) d x_{m+1} \cdots \wedge d x_{n},
$$

we see that

$$
[F]_{s}=c_{n-m}[F] \wedge d \xi_{m+1} \cdots \wedge d \xi_{n}
$$

Note that $d \xi_{i}=d x_{i}^{\#}$ where $d x_{i}$ for $i=m+1, \ldots n$ is a frame for the conormal bundle of $F$. In the next section we shall use this to define superintegration over general smooth submanifolds of $E=\mathbb{R}^{n}$.

An important point to notice is that whereas the standard current of integration is well defined without any extra structure, the supercurrent $[F]_{S}$ depends on the choice of scalar product.

Let $T$ be a supercurrent (or superform) of bidegree $(p, p)$,

$$
T=\sum_{|I|=|J|=p} T_{I J} d x_{I} \wedge d \xi_{J}
$$


Then

$$
\begin{aligned}
J(T) & =\sum_{|I|=|J|=p} T_{I J} d \xi_{I} \wedge d x_{J}(-1)^{p}=\sum_{|I|=|J|=p} T_{I J} d x_{J} \wedge d \xi_{I}(-1)^{p+p^{2}} \\
& =\sum_{|I|=|J|=p} T_{I J} d x_{J} \wedge d \xi_{I} .
\end{aligned}
$$

We therefore say that $T$ is symmetric if $J(T)=T$, or equivalently $T_{I J}=T_{J I}$.

In analogy with the complex case we now say that a symmetric supercurrent $T$ of bidimension $(m, m)$ is (weakly) positive if

$$
T . \alpha_{1} \wedge \alpha_{1}^{\#} \wedge \cdots \alpha_{m} \wedge \alpha_{m}^{\#} \geq 0
$$

for any choice of compactly supported $(1,0)$-forms $\alpha_{j}$. It is then easily verified that the supercurrent of a linear subspace is symmetric and positive, and it is also immediately clear that it is $d$-closed.

Similarly, we say that a superform $\alpha$ of bidegree $(n-m, n-m)$ is (weakly) positive if

$$
\alpha \wedge \alpha_{1} \wedge \alpha_{1}^{\#} \wedge \cdots \alpha_{m} \wedge \alpha_{m}^{\#} \geq 0
$$

at every point. This is clearly equivalent to saying that the expression in (2.6) is positive at any point when $\alpha_{j}$ are constant. When

$$
\alpha=\sum \alpha_{j k} d x_{j} \wedge d \xi_{k}
$$

is of bidegree $(1,1)$, this means that the matrix of coefficients $\left(\alpha_{j k}\right)$ is positive semidefinite. At any point, such a form can be written

$$
\alpha=\sum \alpha_{j} \wedge \alpha_{j}^{\#}
$$

so we get

Proposition 2.1 The wedge product between a positive (symmetric) (1, 1)-form and a positive (symmetric) form of bidegree $(p, p)$ is again positive.

By approximation, the same thing holds for the product of $(1,1)$-forms with currents of bidegree $(p, p)$.

We next introduce the analog of the Kähler form in $\mathbb{R}^{n}$. This is by definition

$$
\beta:=\sum d x_{j} \wedge d \xi_{j}=(1 / 2) d d^{\#}|x|^{2}
$$

Then, the volume form on a linear subspace $F$ can be written

$$
d \lambda_{F}=[F]_{s} \wedge \beta^{m} / m !
$$


in good analogy with the complex case.

In the coming sections we will frequently use contraction with a 1-form in the computations. This is well defined, since we have induced scalar products on the space of all forms, and we define contraction as the dual of exterior multiplication. If $a=\sum a_{j} d x_{j}$ is a $(1,0)$-form, then $\left.a\right\rfloor \beta=a^{\#}=J(a)$, and $\left.a^{\#}\right\rfloor \beta=-a=J\left(a^{\#}\right)$. Therefore,

$$
a\rfloor \beta=J(a)
$$

for any 1-form $a$.

Similarly, contraction with a vector field

$$
\vec{V}=\sum V_{j} \frac{\partial}{\partial x_{j}}
$$

is well defined. Lowering indices, $\vec{V}$ corresponds to the $(1,0)$-form $V=\sum V_{j} d x_{j}$, and $\vec{V}$ and $V$ act in the same way by contraction. Hence, e. g. $\vec{V}\rfloor \beta=V^{\#}$.

Finally, we will discuss how superforms transform under diffeomorphisms: If $G$ is a (local) diffeomorphism on $E=\mathbb{R}^{n}$ and $\alpha=\sum \alpha_{I, J} d x_{I} \wedge d \xi_{J}$ we define the pull back of $\alpha$ under $G$ as

$$
G^{*}(\alpha)=\sum G^{*}\left(\alpha_{I, J} d x_{I}\right) \wedge d \xi_{J}
$$

i. e. the pull back is the standard pull back on the $x$-part of the form and the $d \xi_{j}$ 's are invariant. This means that we extend $G$ to a (local) diffeomorphism on $E_{S}$ by leaving $E_{1}$ fixed, and then take the usual pullback. Then

$$
\int_{\mathbb{R}_{s}^{n}} G^{*}(\alpha)=\int_{\mathbb{R}_{s}^{n}} \alpha
$$

if $\alpha$ is of bidegree $(n, n)$ and $G$ is orientation preserving.

Let now $\vec{V}$ be a vector field on $\mathbb{R}^{n}$ and let $G_{t}$ be its flow, or one parameter family of diffeomorphisms. The classical formula of Cartan for the Lie derivative, Warner (1983), says that if $\alpha$ is of bidegree $(p, 0)$ then

$$
\left.\left.\left.\frac{d G_{t}^{*}(\alpha)}{d t}\right|_{t=0}=d(\vec{V}\rfloor \alpha\right)+\vec{V}\right\rfloor d \alpha=: L_{\vec{V}} \alpha
$$

where $\vec{V}\rfloor$ means contraction with the field $\vec{V}$. This formula holds also for forms of general bidegree. Indeed, this follows immediately from Cartan's formula on the superspace $E_{S}$, if we extend $\vec{V}$ to a vector field on $E_{S}$ that has no component in the second factor. 


\section{Relation to convex functions and tropical varieties}

It is clear from the last part of the previous section that if $\phi$ is a smooth function on $\mathbb{R}^{n}$, then $\phi$ is convex if and only if $d d^{\#} \phi$ is a positive superform. By approximation, it follows that a general, possibly not smooth, function $\phi$ is convex if and only if $d d^{\#} \phi$ is a positive supercurrent. Conversely, if

$$
\alpha=\sum \alpha_{j k} d x_{j} \wedge d \xi_{k}
$$

is a symmetric closed $(1,1)$ current, we can always write $\alpha=d d^{\#} \phi$ for some distribution $\phi$. This is because

$$
\frac{\partial \alpha_{j k}}{\partial x_{l}}=\frac{\partial \alpha_{l k}}{\partial x_{j}}
$$

so

$$
\alpha_{j k}=\frac{\partial \phi_{k}}{\partial x_{j}}
$$

for some $\phi_{k}$. By symmetry

$$
\frac{\partial \phi_{k}}{\partial x_{j}}=\frac{\partial \phi_{j}}{\partial x_{k}}
$$

so $\phi_{k}=\left(\partial \phi / \partial x_{k}\right)$ for some $\phi$. If $\alpha$ is moreover positive, $\phi$ must be convex, so the positive symmetric $(1,1)$-currents are precisely the ones that can be written $d d^{\#} \phi$ for some convex $\phi$.

If $\phi$ is a smooth convex function, we can define $\left(d d^{\#} \phi\right)^{n} / n$ ! which equals

$$
c_{n} \operatorname{det}\left(\frac{\partial^{2} \phi}{\partial x_{j} \partial x_{k}}\right) d x \wedge d \xi
$$

The corresponding measure on $\mathbb{R}^{n}$ is the Monge-Ampére measure of $\phi$

$$
M A(\phi)=\operatorname{det}\left(\frac{\partial^{2} \phi}{\partial x_{j} \partial x_{k}}\right) d x
$$

By the Bedford-Taylor theory, Bedford and Taylor (1976), this definition makes sense for general convex functions. Indeed, Bedford and Taylor define $\left(d d^{c} \phi\right)^{n} / n$ ! for general locally bounded plurisubharmonic functions, hence in particular for convex functions on $\mathbb{R}^{n}$, considered as functions on $\mathbb{C}^{n}$ that do not depend on the imaginary part of the variable. This means that for a general convex function on $\mathbb{R}^{n}$,

$$
\left(d d^{\#} \phi\right)^{n} / n !=M A(\phi)
$$


where the Monge-Ampére measure in the right hand side is taken in the sense of Alexandrov. This follows, since the two sides coincide for smooth functions and are continuous under uniform convergence.

(To be quite honest, Bedford and Taylor (1976) define $\left(d d^{c} \phi\right)^{n}$ as a measure on $\mathbb{C}^{n}$ and prove continuity under decreasing sequences in Bedford and Taylor (1982), hence also for unform convergence when the functions are continuous. Since

$$
\left.\left(d d^{\#} \phi\right)^{n} / n !=\pi_{*}\left(\chi(\xi)\left(d d^{c} \phi\right)^{n} / n !\right)\right),
$$

if $\chi$ is a function of $\xi$ with integral 1 and $\pi$ is the projection from $\mathbb{C}^{n}$ to $\mathbb{R}^{n}$, existence and continuity of $\left(d d^{\#} \phi\right)^{n}$ follows.)

Let us now consider convex functions of the form

$$
\phi(x)=\max _{i \in I} a^{i} \cdot x+b^{i}=\max _{i \in I} l_{i}(x)
$$

where $I$ is a finite set. We will call such functions, i. e. the maxima of a finite collection of affine functions, quasitropical polynomials, reserving the term tropical polynomials for such functions where all components of the (co)vectors $a^{i}$ and the numbers $b^{i}$ are integers, see Mikhalkin (2006). We may assume that all the $a^{i}$ are different. Indeed, if $a^{i}=a^{k}$ and say $b^{i}>b^{k}$, then $l_{i}>l_{k}$ everywhere, and we get the same function if we omit $l_{k}$.

Let $E_{j}=\left\{\phi=l_{j}\right\}$. Then $E_{j}$ is defined by a finite set of linear inequalities. Assume that one of these sets, $E_{k}$ has empty interior. Then we can define

$$
\phi^{k}=\max _{i \neq k} l_{i}
$$

and get a new quasitropical polynomial which equals $\phi$ on a dense set. Hence, by continuity, $\phi_{k}=\phi$ everywhere, so we may as well omit $l_{k}$ in the definition of $\phi$, and can assume that all $E_{j}$ have non empty interior. We therefore get a decomposition of $\mathbb{R}^{n}$ as a finite union of non degenerate but possibly unbounded polyhedra. We then also have that all the $E_{j}$ are different, since if $a^{j} \cdot x+b^{j}=a^{k} \cdot x+b^{k}$ on an open set, $a^{j}=a^{k}$ which we have assumed is not the case.

It is clear that

$$
d^{\#} \phi=\sum \chi_{E_{j}}\left(a^{j}\right)^{\#}
$$

where $\chi_{E_{j}}$ is the characteristic function of the polyhedron $E_{j}$. Since $d \chi_{E_{j}}=\left[\partial E_{j}\right]$ for some choice of orientation, we get

$$
d d^{\#} \phi=\sum\left[F_{l}\right] \wedge v_{l}^{\#}
$$

for some $(1,0)$ (constant) covectors $v_{l}$, where $F_{l}$ is an enumeration of the faces of the polyhedron $E_{j}$. These $v_{l}$ must be normal to $F_{l}$, because $d d^{\#} \phi$ is symmetric (see the next section for this). Positivity of $d d^{\#} \phi$ implies that they point in the same direction as the normal to $F_{l}$ determining the orientation. 
In this way $d d^{\#} \phi$ describes the tropical variety defined by the faces $F_{l}$, endowed with the multiplicity vectors $v_{l}$. (Perhaps it would be more proper to talk of quasitropical variety since the multiplicity vectors are not necessarily integral.) The fact that $d d^{\#} \phi$ is closed is equivalent to the balancing condition in tropical geometry: At a point where several faces intersect, the sum of their multiplicity vectors vanish (see Lagerberg 2012). Conversely, Lagerberg shows that a positive closed supercurrent of bidegree $(1,1)$ with support of dimension $n-1$ (see Lagerberg 2012 for precise, and also more general, statements) equals $d d^{\#} \phi$ for some quasitropical polynomial $\phi$.

\section{Supercurrents associated to general submanifolds of $\mathbb{R}^{n}$}

Let $M$ be a smooth submanifold of $\mathbb{R}^{n}$ of dimension $m$. Given an orientation of $M$ we get the current of integration of $M$. Let us first assume that $M$ is a hypersurface, locally defined by an equation $\rho=0$, where $\rho$ is smooth and has nonvanishing gradient on $M$. Dividing by $|d \rho|$, we may assume that $|d \rho|=1$ on $M$, and we let $n=d \rho$; it is a unit normal form on $M$. Now it is a familiar fact that the current of integration on $M$ can be written

$$
[M]=n * d S_{M}
$$

where $d S_{M}$ is the surface measure on $M$ and the Hodge star indicates that we think of it as a current of degree zero. The choice of sign of $n$ determines the orientation of $M$. From this formula we see in particular that if $[M] \wedge v^{\#}$ is symmetric, then $v$ must be a multiple of $n$, hence normal to $M$ (we used this at the end of the last section, with $M=F_{j}$ ). Moreover, if $[M] \wedge v^{\#}$ is positive, $v$ is a positive multiple of $n$.

Now we define the supercurrent $[M]_{s}$ by

$$
[M]_{s}:=[M] \wedge n^{\#}=n \wedge n^{\#} * d S_{M}
$$

It is clearly positive and symmetric. More generally, if $M$ has codimension $p$, it is locally defined by $p$ equations $\rho_{j}=0$, such that $d \rho_{j}$ are linearly independent on $M$. Replacing $\rho_{j}$ by $\sum a_{j k} \rho_{k}=: \rho_{j}^{\prime}$, for a suitable matrix of functions $a_{j k}$, we may assume that $n_{j}:=d \rho_{j}$ are orthonormal on $M$. Then the currents of integration on $M$ can be written

$$
[M]=n_{1} \wedge \cdots n_{p} * d S_{M}=: n * d S_{M} ; \quad n=n_{1} \wedge \cdots n_{p}
$$

The supercurrent associated to $M$ is defined as

$$
[M]_{s}:=c_{p} n \wedge n^{\#} * d S_{M}
$$

The definition uses the forms $n_{j}$ that are only locally defined, but it is easily verified that a different choice $n_{j}^{\prime}$ leads to the same supercurrent, since $n_{j}$ and $n_{j}^{\prime}$ are related on $M$ by an orthogonal transformation. If $\alpha$ is a superform on the ambient space, 


$$
\alpha \wedge[M]_{s}
$$

kills all the components of $\alpha$ in the directions $n_{j}$ and $n_{j}^{\#}$; we will interpret it as the restriction of $\alpha$ to $M_{s}$; the 'superspace' associated to $M$. Accordingly, we say that $\alpha$ vanishes on $M_{s}$ if (and only if) $\alpha \wedge[M]_{s}=0$

In general, $[M]_{s}$ is not closed, unless $M$ is linear. When computing $d[M]_{S}$ we will have use for the $(1,1)$-forms

$$
F:=d n^{\#}, \quad F_{j}=d n_{j}^{\#}
$$

These forms depend on the choice of defining function(s), but transform in a natural way when we change defining functions. When the codimension $p=1$, the restriction of $F$ to $M$ is the second fundamental form of $M$. In higher codimension the $F_{j}$ restricted to $M$ are the components of the vector valued second fundamental form $\sum F_{j} \otimes \vec{n}_{j}$ with values in the normal bundle of $M$. (Recall that $n_{j}$ form an orthonormal system for the space of normal $(1,0)$ forms at each point.)

Again, we start with the case $p=1$. Then

$$
d[M]_{s}=d\left([M] \wedge n^{\#}\right)=-F \wedge[M],
$$

since $[M]$ is $d$-closed. This expresses $d[M]_{s}$ in terms of $[M]$ but we want to write it in terms of $[M]_{s}$. Therefore we introduce the operator

$$
\left.\mathcal{F}=F \otimes n^{\#}\right\rfloor
$$

which acts on a superform or supercurrent by first contracting with $n^{\#}$ and then wedging with $F$. Notice that $\mathcal{F}$ is an antiderivation. Then

$$
d[M]_{s}=\mathcal{F}[M]_{s}
$$

In a similar way, when $p>1$ we let

$$
\left.\mathcal{F}=\sum_{j} F_{j} \otimes n_{j}^{\#}\right\rfloor,
$$

and still get $d[M]_{s}=\mathcal{F}[M]_{s}$. In the same way we get that

$$
d^{\#}[M]_{s}=-\mathcal{F}^{\#}[M]_{s},
$$

where

$$
\left.\mathcal{F}^{\#}=\sum_{j} F_{j} \otimes n_{j}\right\rfloor .
$$

Because of the following lemma, we can also write $\left.\mathcal{F}=\sum_{j} n_{j}^{\#}\right\rfloor \otimes F_{j}$, i. e. we can first wedge with $F_{j}$ and then contract. 
Lemma $\left.\left.4.1 n_{j}\right\rfloor F_{j}=0=n_{j}^{\#}\right\rfloor F_{j}$ on $M_{s}$, i. e. when wedged with $[M]_{s}$.

Proof We prove this when $p=1$. This is only to simplify the index notation; in the proof $n_{j}$ denotes the components of $n$, i. e. the partial derivatives of $\rho$ with respect to $x_{j}$. Then

$$
n\rfloor F=\sum n_{j} \rho_{j k} d \xi_{k}=1 / 2 \sum \frac{\partial n_{j}^{2}}{\partial x_{k}} d \xi_{k}=1 / 2 d^{\#}|n|^{2} .
$$

Since the norm of $n$ is constant on $M$, this vanishes on $M_{s}$.

\section{Minimal submanifolds}

We start with the following computational proposition.

Proposition 5.1 If $M$ is a smooth m-dimensional submanifold of $\mathbb{R}^{n}$

$$
\left.d\left([M]_{s} \wedge \beta^{p}\right)=\sum_{j} n_{j}^{\#}\right\rfloor\left(F_{j} \wedge[M]_{s} \wedge \beta^{p}\right) .
$$

Proof We have, since $d \beta=0$,

$$
\left.d\left([M]_{s} \wedge \beta^{p}\right)=\sum F_{j} \wedge n_{j}^{\#}\right\rfloor[M]_{s} \wedge \beta^{p} .
$$

Since, by Lemma $\left.4.1, n_{j}^{\#}\right\rfloor\left(F_{j}\right) \wedge[M]_{s}=0$, this equals

$$
\left.\sum n_{j}^{\#}\right\rfloor\left(F_{j} \wedge[M]_{s}\right) \wedge \beta^{p}
$$

Finally, $\left.n_{j}^{\#}\right\rfloor \beta=-n_{j}$, which vanishes when wedged with $[M]_{s}$. This gives the formula in the proposition.

If $F=\sum F_{i j} d x_{i} \wedge d \xi_{j}$ is a $(1,1)$-form,

$$
F \wedge \beta^{n-1} /(n-1) !=\operatorname{tr}(F) \beta^{n} / n !,
$$

where $\operatorname{tr}(F)=\sum F_{i i}$ is the trace of $F$. This implies that

$$
F \wedge[M]_{s} \wedge \beta^{m-1} /(m-1) !=t r^{\prime}(F)[M]_{s} \wedge \beta^{m} / m !
$$

where $\operatorname{tr}^{\prime}(F)$ is the trace of $F$ 's restriction to $M_{s}$, since wedging with $[M]_{s}$ kills all the components of $F$ and $\beta$ in the normal directions. The traces $t r^{\prime}\left(F_{j}\right)=: H_{j}$ are the coefficients of the mean curvature vector

$$
\vec{H}:=\sum H_{j} \vec{n}_{j}
$$


(There seem to be different conventions as to the sign of the mean curvature vector. We follow here the convention in Colding et al. (2015), so that the mean curvature of a sphere points outwards.)

Applying Proposition 5.1 with $p=m-1$ we get

$$
\left.d\left([M] \wedge \beta^{m-1} /(m-1) !\right)=\sum H_{j} n_{j}^{\#}\right\rfloor\left([M]_{s} \wedge \beta^{m} / m !\right) .
$$

Since $n_{j}$ are linearly independent, this vanishes exactly when $\vec{H}$ vanishes, i. e. when $M$ is a minimal manifold, so we have proved

Corollary $5.2[M]_{S} \wedge \beta^{m-1}$ is a closed current if and only if $M$ is a minimal submanifold.

We are therefore led to the following

Definition A positive symmetric supercurrent $T$ of bidimension $(m, m)$ is minimal if

$$
d T \wedge \beta^{m-1}=0
$$

It is clear that this property is conserved by regularisation, e.g. by convolution with an approximate identity. For a general positive supercurrent of bidimension $(m, m)$, if it happens that there is a vector field $\vec{V}=\sum V_{j} \partial / \partial x_{j}$ such that

$$
\left.d T \wedge \beta^{m-1} /(m-1) !=\sum V_{j} d \xi_{j}\right\rfloor\left(T \wedge \beta^{m} / m !\right)
$$

we say that $\vec{V}$ is a mean curvature vector for $T$. As we have seen, this is the case when $T=[M]_{S}$ is associated to a smooth manifold, but it certainly also holds when $T$ is strictly positive and smooth, so that $T \wedge \beta^{m} / m$ ! is a strictly positive volume form. In both these cases, $\vec{V}$ is uniquely determined, so we may speak of the mean curvature vector. In Sect. 8 we shall see that when $T=[M]_{S}$ is associated to a smooth manifold,

$$
d d^{\#}\left(T \wedge \beta^{m-1} /(m-1) !\right)
$$

can be computed in terms of the flow of the mean curvature field.

We next discuss briefly the Dirichlet form on a symmetric and positive supercurrent of bidimension $(m, m)$ :

$$
D(u, u):=\int_{T} d u \wedge d^{\#} u \wedge \beta^{m-1} /(m-1) !=T . d u \wedge d^{\#} u \wedge \beta^{m-1} /(m-1) !,
$$

if $u$ is sufficiently smooth on $\mathbb{R}^{n}$. When $T=[M]_{s}$ is the supercurrent associated to an $m$-dimensional manifold, this is precisely the standard Dirichlet form

$$
\int_{M}\left|d_{M} u\right|^{2} d S_{M}
$$


where $d_{M} u$ is the differential of $u$ restricted to $M$, and $\left|d_{M} u\right|$ is the norm induced by the Euclidean metric on $\mathbb{R}^{n}$. Writing $S_{T}=T \wedge \beta^{m-1} /(m-1)$ ! we have in general

$$
D(u, u)=\int d u \wedge d^{\#} u \wedge S_{T}=-\int u d\left(d^{\#} u \wedge S_{T}\right)
$$

This makes it natural to define the Laplacian on $T$ by

$$
\Delta_{T} u=d\left(d^{\#} u \wedge S_{T}\right)
$$

With this definition, $\Delta_{T} u$ is a measure on $\mathbb{R}^{n}$. If $T$ is the supercurrent of a manifold, or more generally has a mean curvature vector, we can make this more explicit: First we compute

$$
\Delta_{T} u=d d^{\#} u \wedge S_{T}-d^{\#} u \wedge d S_{T}
$$

If $T$ has a mean curvature vector $\mathbf{H}=\sum H_{j} \partial / \partial x_{j}$, and we let $H^{\#}=\sum H_{j} d \xi_{j}$ be the corresponding $(0,1)$ - form, we have with $\sigma=T \wedge \beta^{m} / m$ !,

$$
\left.d S_{T}=H^{\#}\right\rfloor \sigma .
$$

Hence

$$
\left.d^{\#} u \wedge d S_{T}=\left(H^{\#}\right\rfloor d^{\#} u\right) \sigma=\mathbf{H}(u) \sigma .
$$

Therefore

$$
\Delta_{T} u=d d^{\#} u \wedge S_{T}-\mathbf{H}(u) \sigma .
$$

If $T$ is minimal, so that $S_{T}$ is closed and $\mathbf{H}$ vanishes, we have

$$
\Delta_{T} u=d d^{\#} u \wedge S_{T}=d d^{\#} u \wedge \beta^{m-1} /(m-1) ! \wedge T
$$

just like in the Kähler case. In particular, the Laplacian has no first order terms and linear functions are harmonic. For minimal manifolds this is a well known property, cf. Colding and Minicozzi (1999). In general, for $T$ not necessarily minimal, we get by applying (5.9) to a coordinate function, $u=x_{j}$, that the components of $\mathbf{H}, H_{j}=\mathbf{H}\left(x_{j}\right)$ are given by

$$
-H_{j} \sigma=\Delta_{T} x_{j}
$$

(In particular, the mean curvature vector field is uniquely determined.) 


\section{Volume estimates for minimal submanifolds}

To prove volume estimates for minimal manifolds (or supercurrents), we will now follow the method of Lelong to prove such estimates in the complex setting. This requires one little twist since the minimal supercurrent $T$ (e.g. $T=[M]_{S}$ ) is not closed itself; it is only $T \wedge \beta^{m-1}$ that is closed. This is taken care of by the following lemma.

Lemma 6.1 Let for $\delta \geq 0,|x|_{\delta}:=\left(|x|^{2}+\delta\right)^{1 / 2}$, and let for $p>0$

$$
E_{p, \delta}:=\frac{-1}{p}|x|_{\delta}^{-p}
$$

and for $p=0$

$$
E_{0, \delta}=\log |x|_{\delta}
$$

Then, if $p$ is an integer,

$$
d d^{\#} E_{p, \delta} \wedge \beta^{p+1}=\left(d d^{\#}|x|_{\delta}\right)^{p+2} .
$$

Proof This is a direct computation and we will do it for $p>0$, the case $p=0$ being similar but simpler. First,

$$
d^{\#} E_{p, \delta}=|x|_{\delta}^{-(p+2)}(1 / 2) d^{\#}|x|^{2}
$$

and

$$
d d^{\#} E_{p, \delta}=|x|_{\delta}^{-(p+2)}\left(\beta-(p+2) \frac{\gamma}{|x|_{\delta}^{2}}\right),
$$

with $\gamma=d|x|^{2} \wedge d^{\#}|x|^{2} / 4$. On the other hand

$$
d d^{\#}|x|_{\delta}=|x|_{\delta}^{-1}\left(\beta-\frac{\gamma}{|x|_{\delta}^{2}}\right) .
$$

Expanding by the binomial theorem we get

$$
\left(d d^{\#}|x|_{\delta}\right)^{p+2}=|x|_{\delta}^{-(p+2)}\left(\beta^{p+2}-(p+2) \frac{\gamma}{|x|_{\delta}^{2}} \wedge \beta^{p+1}\right) .
$$

The lemma follows from (6.1) and (6.2).

The reason we consider $E_{p, \delta}$ instead of $E_{p, 0}$ is just that we want our functions to be smooth across zero. The main conclusion we draw from the lemma is that

$$
d d^{\#} E_{m-2, \delta} \wedge \beta^{m-1} \wedge T
$$


is positive if $T$ is positive of bidegree $(m, m)$. This follows from Proposition (2.1) since $|x|_{\delta}$ is convex. We also remark that it follows from the proof of the proposition that

$$
d d^{\#} E_{p, \delta} \wedge \beta^{m-1} \wedge T \geq 0
$$

if $0 \leq p \leq m-2$.

Let $T$ be a minimal current of bidimension $(m, m)$, defined in a neighbourhood of the origin. Its mass in a ball of radius $r$ centered at the origin is

$$
\sigma(r):=\int_{|x|<r} T \wedge \beta^{m} / m ! .
$$

In the computations below we first assume that $T$ is smooth. Then, writing $S=$ $T \wedge \beta^{m-1} / m$ !

$$
\begin{aligned}
\sigma(r) & =\int_{|x|=r}(1 / 2) d^{\#}|x|^{2} \wedge S=\int_{|x|=r}|x|_{\delta}^{m} d^{\#} E_{m-2, \delta} \wedge S \\
& =\left(r^{2}+\delta\right)^{m / 2} \int_{|x|<r}\left(d d^{\#}|x|_{\delta}\right)^{m} \wedge T / m !
\end{aligned}
$$

since $d S=0$. Since the integrand in the right hand side is nonnegative it follows that

$$
\left(r^{2}+\delta\right)^{-(m / 2)} \sigma(r)
$$

is (weakly) increasing. This holds for any $\delta>0$, so $r^{-m} \sigma(r)$ is also increasing. By approximation with smooth forms, this holds also for general minimal currents, so we have proved the following generalization of the monotonicity theorem for minimal manifolds (see Colding and Minicozzi 1999).

Theorem 6.2 Let $T$ be a minimal (super)current of bidimension $(m, m)$ defined in a neighbourhood of the origin. Then

$$
r^{-m} \int_{|x|<r} T \wedge \beta^{m} / m !
$$

is nondecreasing.

When $T$ is the supercurrent of a minimal manifold, this says that the area of the manifold inside a ball of radius $r$, divided by $r^{-m}$ is nondecreasing. In analogy with the case of minimal manifolds we call

$$
\gamma_{T}(0):=\lim _{r \rightarrow 0} r^{-m} \int_{|x|<r} T \wedge \beta^{m} / m !,
$$

the density of $T$ at the origin. When $T$ is the supercurrent of a (smooth) minimal manifold it equals $\omega_{m}$, the volume of $m$-dimensional unit ball. (The corresponding 
limit for closed positive $(m, m)$-currents in complex analysis is the Lelong number of the current.)

Let us now look again at the formula

$$
\int_{|x|<r} T \wedge \beta^{m} / m !=\left(r^{2}+\delta\right)^{m / 2} \int_{|x|<r} T \wedge\left(d d^{\#}|x|_{\delta}\right)^{m} / m ! .
$$

We proved this for $T$ smooth, but by approximation it holds for general minimal supercurrents of bidimension $(m, m)$. It means in particular that the integral in the right hand side is bounded as $\delta \rightarrow 0$, so there is a subsequence of $\delta$ 's such that

$$
T \wedge\left(d d^{\#}|x|_{\delta}\right)^{m} / m !
$$

converges weakly to a measure, $\mu$. Outside the origin, where $|x|$ is smooth, this measure must be

$$
T \wedge\left(d d^{\#}|x|\right)^{m} / m !
$$

We also see that $\mu$ must have a point mass at the origin of size $\gamma_{T}(0)$, since the mass of $\mu$ in any ball centered at the origin with small radius $r$ is

$$
r^{-m} \int_{|x|<r} T \wedge \beta^{m} / m !
$$

Thus, every subsequence has the same limit

$$
\mu=\chi_{x \neq 0} T \wedge\left(d d^{\#}|x|\right)^{m} / m !+\gamma_{T}(0) \delta_{0} .
$$

By Lemma 6.1 we also have

$$
\lim _{\delta \rightarrow 0} T \wedge d d^{\#} E_{m-2, \delta} \wedge \beta^{m-1} / m !=\mu,
$$

which can be interpreted as saying that the 'Laplacian' of $E_{m-2,0}$ on $T$ (the trace of $d d^{\#} E_{m-2,0}$ ) equals a point mass at the origin of size $\gamma_{T}$, plus a nonnegative contribution outside the origin. The contribution outside the origin vanishes when $T$ is the supercurrent of an $m$-dimensional plane through the origin. This reflects the fact that $E_{m-2,0}$ is a fundamental solution of the Laplacian then; the crucial observation is that $E_{m-2,0}$ is always 'subharmonic on $T$ '.

We shall next generalize the proof of Theorem 6.2 to a general domain, $D$. Then $|x|$ is not constant on the boundary of $D$, so instead we write $|x|^{m}=w(x)$ on the boundary, where $w$ is a positive smooth function on the closure of $D$ to be chosen later. Let $T$ be a minimal supercurrent (which we tacitly take as smooth at first) in a neighbourhood of $\bar{D}$. Then the mass of $T$ in $D$ is

$$
\int_{D} T \wedge \beta^{m} / m !=\int_{\partial D} d^{\#}|x|^{2} / 2 \wedge S / m !=\int_{\partial D} w d^{\#} E_{m-2} \wedge S / m !
$$


(Here we skip the part of the argument where we approximate $|x|$ by $|x|_{\delta}$ and we write $E_{m-2}$ for $E_{m-2,0}$.) By Stokes' theorem this equals

$$
\int_{D} w d d^{\#} E_{m-2} \wedge S / m !+\int_{D} d w \wedge d^{\#} E_{m-2} \wedge S / m !=: I+I I
$$

since $S$ is closed. By what we have just seen, $I \geq w(0) \gamma_{T}(0)$. To see when $I I$ is positive we compute (for $m>2$ )

$$
\begin{aligned}
0= & (m-2)^{-1} \int_{\partial D}\left(\frac{1}{|x|^{m-2}}-\frac{1}{w^{1-2 / m}}\right) d^{\#} w \wedge S \\
= & \frac{1}{m-2} \int_{D}\left(\frac{1}{|x|^{m-2}}-\frac{1}{w^{1-2 / m}}\right) d d^{\#} w \wedge S+m^{-1} \int_{D} \frac{1}{w^{2-2 / m}} d w \wedge d^{\#} w \wedge S \\
& -\int_{D} d E_{m-2} \wedge d^{\#} w \wedge S .
\end{aligned}
$$

Since

$$
d \phi \wedge d^{\#} \psi \wedge S=d \psi \wedge d^{\#} \phi \wedge S
$$

when $S$ is symmetric we find that

$$
\begin{aligned}
I I= & \frac{1}{m-2} \int_{D}\left(\frac{1}{|x|^{m-2}}-\frac{1}{w^{1-2 / m}}\right) d d^{\#} w \wedge S / m ! \\
& +m^{-1} \int_{D} \frac{1}{w^{2-2 / m}} d w \wedge d^{\#} w \wedge S / m !
\end{aligned}
$$

It follows that $I I \geq 0$ if $w \geq|x|^{m}$ in $D$ and $w$ is convex.

Theorem 6.3 Let $D$ be a smoothly bounded domain in $\mathbb{R}^{n}$. Let a be any point in $D$ and let $w$ be a convex function on $\bar{D}$ such that $w=|x-a|^{m}$ on the boundary of $D$. If $T$ is a minimal supercurrent in $D$ of bidimension $(m, m)$, then its mass satisfies

$$
\int_{D} T \wedge \beta^{m} / m ! \geq w(a) \gamma_{T}(a)
$$

(where $\gamma_{T}(a)$ is the density of $T$ at a).

(We shall see in the next section that the convexity assumption on $w$ can be relaxed considerably.)

In the proof we may of course assume that $a=0$. For $m>2$ the theorem follows immediately from the argument above, since we can always assume that $w \geq|x|^{m}$, replacing it if necessary by $\max \left(w,|x|^{m}\right)$. The case $m=2$ is similar; we just have to replace the boundary integral in $(6.5)$ by

$$
\int_{\partial D} \log \frac{w}{|x|^{2}} d^{\#} w \wedge S
$$


As a special case we get a recent result of Brendle and Hung (2017), which generalizes an older estimate of Alexander and Osserman for the case $m=2$ (Alexander and Osserman 1975).

Corollary 6.4 Let $M$ be a minimal manifold of dimension $m$ in the unit ball which contains the point $a$. Then the volume of $M$ satisfies

$$
|M| \geq \omega_{m}\left(1-|a|^{2}\right)^{m / 2},
$$

where $\omega_{m}$ is the volume of the m-dimensional unit ball.

Proof We have

$$
|x-a|^{2}=1+|a|^{2}-2 a \cdot x=: v
$$

on the boundary of the ball. Since $v$ is convex (in fact linear), $w:=v^{m / 2}$ is also convex and the corollary follows directly from the theorem, since $\gamma_{T}(a)=\omega_{m}$ when $T$ is the supercurrent of a (smooth) manifold.

\section{Removable singularities}

The techniques of the previous section can also be used to give a variant of the El Mir-Skoda theorem on extension of positive closed currents (El Mir 1984; Skoda 1982) in the setting of minimal manifolds or minimal currents. It should be stressed that here we are not aiming to prove that a minimal surface with possible singularities on a small set is actually smooth, but rather that the minimal current defined by the surface outside the singularities extends as a minimal current. This is a much simpler problem than regularity, but the results are still useful as they will allow us to apply the calculus of supercurrents to manifolds with singularities on a set of sufficiently small dimension. We refer to Harvey and Lawson (1975) for a discussion of and very precise results on removable singularities in the classical setting.

We first define a locally integrable function $u$ to be $m$-subharmonic if

$$
d d^{\#} u \wedge \beta^{m-1}
$$

is positive. This condition is preserved under regularization (convolution with an approximate identity) so we can approximate general $m$-subharmonic functions with smooth ones. If $u$ is smooth and we fix a point we can make an orthogonal change of coordinates so that

$$
d d^{\#} u=\sum \lambda_{j} d x_{j} \wedge d \xi_{j}
$$

at that point. We also have that

$$
\beta^{m-1} /(m-1) !=\sum_{|I|=m-1} d V_{I},
$$


where

$$
d V_{I}=d x_{i_{1}} \wedge d \xi_{i_{1}} \wedge \cdots d x_{i_{m-1}} \wedge d \xi_{i_{m-1}}
$$

for $I=\left(i_{1}, \ldots i_{m-1}\right)$. Thus, at the chosen point,

$$
d d^{\#} u \wedge \beta^{m-1} /(m-1) !=\sum_{|J|=m} \lambda_{J} d V_{J},
$$

where $\lambda_{J}=\sum_{i \in J} \lambda_{i}$. In conclusion, a smooth function $u$ is $m$-subharmonic if and only if the sum of any $m$-tuple of eigenvalues of $d d^{\#} u$ is nonnegative at any point. We also see from (7.1) that if $u$ is smooth and $m$-subharmonic, then

$$
d d^{\#} u \wedge \beta^{m-1} \wedge T \geq 0
$$

if $T$ is a positive supercurrent of bidegree $(n-m, n-m)$.

Lemma 6.1 implies that $E_{m-2, \delta}$ is $m$-subharmonic, and taking limits when $\delta \rightarrow 0$ the same thing holds for $E_{m-2}$. Therefore, any potential

$$
u(x):=\int \frac{-1}{|x-y|^{m-2}} d \mu(y)
$$

for $m>2$ and

$$
u(x):=\int \log |x-y| d \mu(y)
$$

when $m=2$ is also $m$-subharmonic, if $\mu$ is a positive measure.

Kernels like $E_{m-2}$ on $\mathbb{R}^{n}$ are called Riesz kernels, and have a well developed potential theory, following the lines of the more classical potential theory for the Newtonian kernel $E_{n-2}$, see Landkof (1972). Thus, we have a notion of capacity $C_{m-2}$ associated to $E_{m-2}$ and any set of sigma-finite $(m-2)$-dimensional Hausdorff measure has capacity zero.

We also say that a set $F$ is $m$-polar is there is an $m$-subharmonic function which is equal to $-\infty$ on $F$ (and maybe elsewhere as well). By Landkof (1972), any compact set $K$ with $C_{m-2}(K)=0$ is $m$-polar, and moreover there is a measure $\mu$ supported on $K$ whose potential equals $-\infty$ precisely on $K$. Therefore we get

Proposition 7.1 Any compact set $K$ of $\sigma$-finite $(m-2)$-dimensional Hausdorffmeasure is $m$-polar and there is a potential $u$ of a measure supported on $K$ which equals $-\infty$ on $K$.

To illustrate this, notice that it is immediate that a discrete set of points is 2-polar, and that a submanifold of dimension $(m-2)$ is $m$-polar in general. Indeed, it suffices to take $\mu$ equal to surface measure. 
Theorem 7.2 Let $T$ be a minimal supercurrent of bidimension $(m, m)$, defined in $B \backslash K$, where $B$ is a ball and $K$ and is compact in $\mathbb{R}^{n}$ with sigma-finite $(m-2)$-dimensional Hausdorff measure. Assume that T has finite mass

$$
\int_{B \backslash K} T \wedge \beta^{m}<\infty
$$

Then the trivial extension of $T, \tilde{T}:=\chi_{B \backslash K} T$, is a minimal supercurrent in $B$.

Proof We follow almost verbatim the proof of Proposition 11.1 in Berndtsson and Sibony (2002). Let $u$ be a potential as in (7.2) with $\mu$ supported on $K$, which equals $-\infty$ on $K$. Thus, $u$ is smooth outside $K$ and $m$-subharmonic. Let $\chi(t)$ be an increasing smooth convex function, defined when $t \leq 0$, with $\chi(0)=1$ and $\chi(t)=0$ for $t \leq-1$. Put

$$
u_{k}=\chi(u / k)
$$

for $k=1,2, \ldots$. Then $u_{k}$ are smooth, $m$-subharmonic, $0 \leq u_{k}<1$, and $u_{k}$ tend to 1 uniformly on compacts outside $K$. Moreover, all $u_{k}$ vanish in a neighbourhood of $K$.

Let $\theta$ be a smooth function with compact support in $B$. Then, by integration by parts,

$$
\int_{B} \theta T \wedge \beta^{m-1} \wedge d d^{\#} u_{k}^{2}=\int_{B} u_{k}^{2} d d^{\#} \theta \wedge T \wedge \beta^{m-1} \leq C
$$

where $C$ is a fixed constant independent of $k$. Here we have used that $T \wedge \beta^{m-1}$ is closed outside of $K$, and that $u_{k}$ vanishes near $K$. This implies that

$$
\int_{B} \theta T \wedge \beta^{m-1} \wedge d u_{k} \wedge d^{\#} u_{k} \leq C
$$

since

$$
d d^{\#} u_{k}^{2}=2 u_{k} d d^{\#} u_{k}+2 d u_{k} \wedge d^{\#} u_{k}
$$

and $d d^{\#} u_{k} \wedge \beta^{m-1} \geq 0$ for $u_{k} m$-subharmonic.

Let $p(x)$ be a smooth function on $\mathbb{R}$, such that $p(x)=1$ if $x>1 / 2$ and $p(x)=0$ if $x<1 / 3$, and put $\chi_{k}=p\left(u_{k}\right)$. Then $\chi_{k}$ tends to $\chi_{B \backslash K}$ and we get if $\psi$ is a smooth $(1,0)$-form supported in $B$,

$$
\begin{aligned}
\int_{B} d\left(\chi_{B \backslash K} T \wedge \beta^{m-1}\right) \wedge \psi^{\#} & =\lim _{k \rightarrow \infty} \int_{B} d\left(\chi_{k} T \wedge \beta^{m-1}\right) \wedge \psi^{\#} \\
& =\lim _{k \rightarrow \infty} \int_{B} p^{\prime}\left(u_{k}\right) d u_{k} \wedge T \wedge \beta^{m-1} \wedge \psi^{\#}
\end{aligned}
$$


The integral

$$
\int_{B \backslash K} \alpha \wedge \tau^{\#} \wedge T \wedge \beta^{m-1}=:(\alpha, \tau)
$$

is a positive semidefinite bilinear form on the space of smooth $(1,0)$-forms in $B$. By Cauchy's inequality we have, choosing $\alpha=d u_{k}$ and $\tau=p^{\prime}\left(u_{k}\right) \psi$ that

$$
\begin{aligned}
& \int_{B} p^{\prime}\left(u_{k}\right) d u_{k} \wedge T \wedge \beta^{m-1} \wedge \psi^{\#} \\
& \leq \sqrt{\int_{B \backslash K} d u_{k} \wedge d^{\#} u_{k} \wedge T \wedge \beta^{m-1} \int_{B \backslash K}\left(p^{\prime}\left(u_{k}\right)\right)^{2} \psi \wedge \psi^{\#} \wedge T \wedge \beta^{m-1}}
\end{aligned}
$$

(It does not matter if we integrate over $B$ or $B \backslash K$ in the left hand side since $d u_{k}$ and $p^{\prime}\left(u_{k}\right)$ vanish near $K$.) By (7.3), the first integral on the right hand side is bounded independently of $k$. The second integral in the right hand tends to zero by dominated convergence since $p^{\prime}\left(u_{k}\right)$ tends to zero on $B \backslash K$. Hence

$$
\lim _{k \rightarrow \infty} \int_{B} p^{\prime}\left(u_{k}\right) d u_{k} \wedge T \wedge \beta^{m-1} \wedge \psi^{\#}=0
$$

Therefore $\chi_{B \backslash K} T \wedge \beta^{m-1}$ is $d$-closed, so we are done.

One consequence of this is that Corollary 6.4 also holds for minimal manifolds with singularities. More precisely, by the theorem, the extension $\tilde{T}$ has a density also at points in $K$. It is easy to see, by the monotonicity theorem, that

$$
\gamma_{\tilde{T}}(a) \geq \limsup _{b \rightarrow a} \gamma_{\tilde{T}}(b)
$$

If $T$ is associated with a minimal manifold of dimension $m$ that has singularities at the set $E$ the density is $\omega_{m}$ (the volume of an $m$-dimensional unit ball) at all the regular points. Therefore it is at least $\omega_{m}$ at the singular points.

(A natural question in this context seems to be if minimal manifolds can be characterized within the class of minimal supercurrents by conditions on the density. For instance, if $T$ is minimal and the density is constant on the support of $T$, is $T$ then $c[M]_{s}$ for a smooth minimal manifold $M$ ?)

We also note that the assumption that $w$ be convex in Theorem 6.3 can be relaxed to $m$-subharmonic, since we just need that

$$
d d^{\#} w \wedge S=d d^{\#} w \wedge T \wedge \beta^{m-1}
$$

be nonnegative. 


\section{Variation of volume and the mean curvature flow}

We have seen in formula (5.1), that if $M$ is a smooth manifold of dimension $m$, then

$$
\left.d\left([M]_{s} \wedge \beta^{m-1} /(m-1) !\right)=\sum H_{j} n_{j}^{\#}\right\rfloor\left([M]_{s} \wedge \beta^{m} / m !\right)
$$

where $H_{j}$ are the components of the mean curvature vector. In this section we will use the notation $\beta_{m}=\beta^{m} / m$ !,

$$
S=[M]_{s} \wedge \beta_{m-1},
$$

and

$$
\sigma=[M]_{s} \wedge \beta_{m}
$$

Then formula (8.1) says that

$$
\left.d S=H^{\#}\right\rfloor \sigma
$$

Since $S$ is $J$-invariant and of even degree it follows after a small computation that

$$
\left.d^{\#} S=J d S=-H\right\rfloor \sigma
$$

Our main objective in this section is to give a formula for the derivative of the volume form

$$
\sigma_{M}:=[M]_{s} \wedge \beta^{m} / m !
$$

when $M$ moves under the mean curvature flow, see Colding et al. (2015), Smoczyk (1999). As in these references, we say that a family $M_{t}$ of smooth submanifolds of $\mathbb{R}^{n}$ moves by the mean curvature flow if there is a vector field $\vec{H}$ on the ambient space such that $F_{t}\left(M_{0}\right)=M_{t}$, where $F_{t}$ is the flow of $-\vec{H}$, which restricts to the mean curvature vector field of $M_{t}$ on each $M_{t}$.

Again we point out that we use here the same conventions about the sign of the mean curvature vector as in Colding et al. (2015), which seems to be the opposite of the one in Smoczyk (1999). To make matters worse, the condition

$$
F_{t}\left(M_{0}\right)=M_{t}
$$

means in terms of pullbacks of currents that

$$
F_{-t}^{*}\left(\left[M_{0}\right]\right)=\left[M_{t}\right]
$$

so since we will work with pullbacks, we are dealing with the flow $G_{t}$ of the vector field $+\vec{H}$ after all. Recalling Cartan's formula for the Lie derivative (cf. Sect. 2) we get 


$$
\left.\left.\left.\frac{d}{d t}\right|_{t=0}\left[M_{t}\right]=L_{\vec{H}}[M]=d(\vec{H}\rfloor[M]\right)=d(H\rfloor[M]\right),
$$

since $[M]$ is closed.

We first note that the next proposition follows directly from (8.3) and Cartan's formula.

\section{Proposition 8.1}

$$
\left.\frac{d F_{-t}^{*}(\sigma)}{d t}\right|_{t=0}=-d d^{\#} S
$$

We now let $\sigma_{t}=\left[M_{t}\right]_{s} \wedge \beta_{m}$; the volume form of $M_{t}$. (Note that this is not the same thing as $F_{-t}^{*}(\sigma)$ which has total mass independent of $t$, whereas the mass of $M_{t}$ changes.) The next theorem is the main result of this section.

Theorem 8.2 Let $M_{t}$ be a family of smooth m-dimensional submanifolds of $\mathbb{R}^{n}$, moving under the mean curvature flow, with $M_{0}=M$. Let

$$
\sigma_{t}=\sigma_{M_{t}}=\left[M_{t}\right]_{s} \wedge \beta_{m}
$$

be their volume forms. Then

$$
\left.\frac{d \sigma_{t}}{d t}\right|_{t=0}=-|\vec{H}|^{2} \sigma-d d^{\#} S
$$

We will deduce the theorem from the proposition. Note that

$$
F_{-t}^{*}(\sigma)=F_{-t}^{*}\left(\left[M_{0}\right]_{s}\right) \wedge F_{-t}^{*}\left(\beta_{m}\right) .
$$

We now differentiate this with respect to $t$ at $t=0$ and use the proposition in the left hand side. The result is

$$
-d d^{\#} S=\left.\frac{d \sigma_{t}}{d t}\right|_{t=0}+\left.\left[M_{0}\right]_{s} \wedge \frac{d}{d t}\right|_{t=0} F_{-t}^{*}\left(\beta_{m}\right) .
$$

By Cartan's formula

$$
\left.\left.\frac{d}{d t}\right|_{t=0} F_{-t}^{*}\left(\beta_{m}\right)=d(H\rfloor \beta\right) \wedge \beta_{m-1}=d H^{\#} \wedge \beta_{m-1} .
$$

Hence

$$
\left.\left[M_{0}\right]_{s} \wedge \frac{d}{d t}\right|_{t=0} F_{-t}^{*}\left(\beta_{m}\right)=\left(d H^{\#}\right) \wedge\left[M_{0}\right]_{s} \wedge \beta_{m-1}=d\left(H^{\#} \wedge S\right)+H^{\#} \wedge d S .
$$

But

$$
H^{\#} \wedge S=0
$$


since $H^{\#}=\sum H_{j} n_{j}^{\#}$ and $S$ contains a factor $n^{\#}=n_{1}^{\#} \wedge \ldots n_{p}^{\#}$. Moreover, by (8.2),

$$
\left.H^{\#} \wedge d S=H^{\#} \wedge\left(H^{\#}\right\rfloor \sigma\right)=|H|^{2} \sigma .
$$

Thus

$$
\left.\left[M_{0}\right]_{s} \wedge \frac{d}{d t}\right|_{t=0} F_{-t}^{*}\left(\beta_{m}\right)=|H|^{2} \sigma
$$

and inserting this in (8.6) the theorem follows.

Integrating (8.5) against a smooth function $\rho$, and using (2.4), we obtain

Corollary 8.3 Let $M_{t}$ be a family of compact manifolds of dimension $m$, moving under the mean curvature flow. Then, if $\rho$ is a sufficiently smooth function

$$
\left.(d / d t)\right|_{t=0} \int \rho \sigma_{t}=-\int \rho|\vec{H}|^{2} \sigma-\int d d^{\#} \rho \wedge S .
$$

As a consequence, if $\rho$ is nonnegative and convex (or, more generally, $m$-subharmonic), then

$$
\int \rho \sigma_{t}
$$

is decreasing.

To understand the intuitive meaning of the corollary, let first $\rho$ be constant equal to 1 . Then we get the well known fact that the volume of $M$ decreases under the mean curvature flow. When $\rho$ is nonconstant, convex and nonnegative we get an extra negative contribution to the derivative of the integral of $\rho$ over $M_{t}$, so, roughly speaking, $M_{t}$ tends to move towards where $\rho$ is small. The next consequence is a more precise formulation of this.

Corollary 8.4 Under the same hypotheses as in Theorem 8.2, assume also that $M=M_{0}$ lies in a convex open set $\Omega$. Then $M_{t}$ lies in $\Omega$ for $t>0$ as long as the mean curvature flow exists.

This follows since we may choose $\rho$ to be zero in the convex set and strictly positive outside. Gerhard Huisken has kindly informed me that this is a well known property that follows from the parabolic maximum principle, using the formula for the mean curvature field in formula (5.11).

An open set is convex if it has a convex defining function, $i$. e. if there is a nonnegative convex function $\rho$, such that $\Omega=\{\rho<1\}$. Analogously we could define $m$-convex domains as domains that possess an $m$-subharmonic defining function. (Recall that we have defined $\rho$ to be $m$-subharmonic if $d d^{\#} \rho \wedge \beta_{m-1} \geq 0$. ) Then the last corollary also holds if $\Omega$ is only $m$-convex, where $m$ is the dimension of $M$. This condition becomes weaker when the dimension is large: For curves we need convexity, but for hypersurfaces $(n-1)$-convexity is enough. 
Formula (8.5) reflects the fact that the mean curvature flow is described by a (non linear) parabolic equation. It says that the volume forms then flow by a (linear) parabolic equation. It seems to be a natural question if there is a corresponding equation for the supercurrents $\left[M_{t}\right]_{s}$ themselves, i. e. without taking traces as we have done here. Since the minimal surface equation becomes linear in the supercurrents formalism, this is perhaps not completely unrealistic.

\section{General submanifolds and their Riemannian geometry}

In this section we shall describe the Levi-Civita connection and Riemannian curvature of a submanifold $M$ of $\mathbb{R}^{n}$ in terms of the 'superstructure'.

Let $M$ be a smooth submanifold of $\mathbb{R}^{n}$ and let $[M]_{s}$ be its associated supercurrent. With the notation from Sect. 3 we have

$$
d[M]_{s}=\mathcal{F}[M]_{s}, \quad d^{\#}[M]_{s}=-\mathcal{F}^{\#}[M]_{s} .
$$

Motivated by these formulas we introduce the operators

$$
D:=d-\mathcal{F}, \quad D^{\#}: d^{\#}+\mathcal{F}^{\#}
$$

and get

$$
D[M]_{s}=D^{\#}[M]_{s}=0 .
$$

Notice that $D$ and $D^{\#}$ are antiderivations, so they satisfy the same rules of computation as $d$ and $d^{\#}$. If $a$ is a superform on the ambient space we get

$$
D\left(a \wedge[M]_{s}\right)=(D a) \wedge[M]_{s}, \quad D^{\#}\left(a \wedge[M]_{s}\right)=\left(D^{\#} a\right) \wedge[M]_{s} .
$$

In particular, if $a$ vanishes on $[M]_{s}$, i.e. $a \wedge[M]_{s}=0$, then $D a$ and $D^{\#} a$ also vanish on $[M]_{s}$, which means that $D$ and $D^{\#}$ are well defined as operators on forms on $[M]_{s}$.

Since $\mathcal{F}$ involves contraction with $n_{j}^{\#}$, it vanishes on any form of bidegree $(p, 0)$. Hence $D=d$ on such forms and we have just retrieved the fact that the exterior derivative is well defined on submanifolds. On the other hand, $\mathcal{F}^{\#}$ involves contraction with $n_{j}$ and does not vanish on forms of bidegree $(p, 0)$, so $d^{\#}$ and $D^{\#}$ are different on $(p, 0)$ forms. In fact, $D^{\#} a$ can be thought of as the Levi-Civita connection acting on $a$. Indeed, $D^{\#} a$ is a form of bidegree $(p, 1)$, so it can be seen as a 1 -form in $\xi$ with values in the space of $(p, 0)$-forms in $x$. If $V=\sum V_{j} \partial / \partial x_{j}$ is a tangent vector to $M$, the derivative of $a$ in the direction $V$ is

$$
\left.V^{\#}\right\rfloor D^{\#} a,
$$

where $V^{\#}=\sum V_{j} \partial / \partial \xi_{j}$. It is not hard to verify that this is the Levi-Civita connection. 
To compute its curvature we need to apply $D^{\#}$ twice. This is done in the following proposition which is just a way of writing Gauß's formula for the curvature in supernotation (see also Weyl (1939)).

Proposition 9.1 The curvature tensor $R$ on $M$ is given by the symmetric $(2,2)$ form

$$
R=(1 / 2) \sum F_{j} \wedge F_{j}
$$

in the sense that, if a is of bidegree $(1,0)$

$$
\left.\left(D^{\#}\right)^{2} a=a\right\rfloor(1 / 2) \sum F_{j} \wedge F_{j}
$$

If $a$ is a form of bidegree $(p, q)$ on $M_{S}$ we can always extend it to a $(p, q)$-form on the ambient space. It is convenient to choose a special extension. We say that the extension is canonical if $\left.\left.n_{j}\right\rfloor a=n_{j}^{\#}\right\rfloor a=0$ for $j=1, \ldots p$. It is obvious that canonical extensions exist locally since $n_{j}=d \rho_{j}$ are linearly independent and can be completed to a basis for the $(1,0)$ forms. We then just take any extension, write it in terms of the new basis, and throw away the terms that contain some $n_{j}$ or $n_{j}^{\#}$. The resulting form coincides with $a$ on $M_{S}$ in the sense that its wedge product with $[M]_{S}$ equals $a \wedge[M]_{s}$.

If $a$ is a canonical extension of a form on $M_{s}$, then $\mathcal{F} a=\mathcal{F}^{\#} a=0$, so

$$
D^{\#} a=d^{\#} a \text {. }
$$

Thus

$$
\left.\left(D^{\#}\right)^{2} a=\mathcal{F}^{\#} d^{\#} a=\sum F_{j} \wedge n_{j}\right\rfloor d^{\#} a
$$

To continue, we introduce the notation that if $F=\sum F_{i j} d x_{i} \wedge d \xi_{j}$ is a $(1,1)$ form (on $\left.\mathbb{R}_{s}^{n}\right)$ and $a$ is $(p, q)$ form, then

$$
\left.F \cup a=-\sum F_{i j} d \xi_{j} \wedge\left(d x_{i}\right\rfloor a\right)
$$

Notice that in the particular case when $a$ has bidegree $(1,0)$

$$
F \cup a=-a\rfloor F
$$

Lemma 9.2 If $F=d \theta^{\#}$ (where $\theta$ is $\left.(1,0)\right)$, then we have the commutator formula

$$
\left.\left.\left.\left[d^{\#}, \theta\right\rfloor\right] a:=d^{\#} \theta\right\rfloor a+\theta\right\rfloor d^{\#} a=-F \cup a
$$

where $a$ is any superform (canonical or not).

Proof First we note that the commutator is a scalar operator, so that

$$
\left.\left.\left[d^{\#}, \theta\right\rfloor\right] f a=f\left[d^{\#}, \theta\right\rfloor\right] a
$$


if $f$ is a function. Therefore we may assume that $a=d x_{I} \wedge d \xi_{J}$. Then $d^{\#} a=0$ and

$$
\left.\left.d^{\#} \theta\right\rfloor a=\sum \frac{\partial \theta_{i}}{\partial x_{j}} d \xi_{j} \wedge\left(d x_{i}\right\rfloor a\right),
$$

which proves the formula.

The lemma gives that

$$
\left(D^{\#}\right)^{2} a=-\sum F_{j} \wedge F_{j} \cup a,
$$

for $a$ of any bidegree. Combined with the remark immediately before the lemma, this gives that

$$
\left.\left(D^{\#}\right)^{2} a=a\right\rfloor(1 / 2) \sum F_{j} \wedge F_{j}
$$

if $a$ is $(1,0)$, which concludes the proof of the proposition.

From Proposition 9.1 we see that integrals like

$$
\int_{[M]_{s}} \chi\left(\sum F_{j} \wedge F_{j}\right)^{q} \wedge \beta^{m-2 q}
$$

where $\chi$ is a function on $M$ are intrinsic, i. e. they do not depend on the embedding of $M$ in $\mathbb{R}^{n}$, since the Riemann curvature (and the metric) are intrinsic. (This is of course not true for similar integrals containing arbitrary combinations of $F_{j}$.) In the next section we shall give an illustration of this.

\section{Weyl's tube formula}

We will now use the formalism of the previous section to give a quick proof of Weyl's tube formula (Weyl 1922; Gray 1990). The proof is not really different from the original proof, but the formalism helps to organise the formulas.

Let $M$ be a compact submanifold of $\mathbb{R}^{n}$. We will consider $T_{r}(M)$, the tube around $M$ of width $r$, which when $M$ is without boundary is defined as

$$
T_{r}(M)=\left\{x \in \mathbb{R}^{n} ; d(x, M)<r\right\} .
$$

When $r$ is sufficiently small, $T_{r}(M)$ is by the tubular neighbourhood theorem diffeomorphic to a neighbourhood of the zero section of the normal bundle $N(M)$ of M,

$$
\Delta(M, r):=\{v \in N(M) ;|v|<r\} .
$$


A point $v$ in the normal bundle is a vector, normal to $T_{p}(M)$ where $p=\pi(v), \pi$ being the projection from $N(M)$ to $M$, and the diffeomorphism is

$$
G: \Delta(M, r) \rightarrow \mathbb{R}^{n}, \quad G(v)=\pi(v)+v .
$$

When $M$ is a compact manifold with boundary, we define $T_{r}(M)$ to be the image of $\Delta(M, r)$ under this map. Weyl's tube formula expresses the volume of these tubes, $\left|T_{r}(M)\right|$, in terms of curvature integrals over the manifold.

Theorem 10.1 Let $M$ be a compact $m$-dimensional submanifold of $\mathbb{R}^{n}$ and let $T_{r}(M)$ be the tube of width $r$ around $M$. Then, for $r$ small

$$
\left|T_{r}(M)\right|=\sum_{2 q \leq m} c_{2 q} r^{2 q+p} \int_{[M]_{s}} R^{q} \wedge \beta^{m-2 q}
$$

for some constants $c_{2 q}$ that can be explicitly calculated, where $p=n-m$ is the codimension of $M$.

Thus the theorem says first that the volume of the tubes of width $r$ is a polynomial in $r$ for $r$ small, and moreover and most remarkably that the coefficients of the polynomial are intrinsic. Thus, if we have two isometric embeddings of the Riemannian manifold $M$ into $\mathbb{R}^{n}$, the tube volumes are the same.

For the proof, we have first

$$
\left|T_{r}(M)\right|=\int_{\left[T_{r}(M)\right]_{s}}\left(\sum d x_{k} \wedge d \xi_{k}\right)^{n} / n ! .
$$

It is enough to prove the theorem locally; i. e. we may assume that the normal forms $n_{j}=d \rho_{j}$ of Sect. 4 are defined on all of $M$. Then the normal bundle is trivial and isometric to $M \times B_{r}(0)$, where $B_{r}(0)$ is the ball in $\mathbb{R}^{n}$ of radius $r$ and center 0 , via the orthonormal frame $\vec{n}_{j}$. Hence the diffeomorphism $G$ described above can be written

$$
G: M \times B_{r}(0) \rightarrow T_{r}(M)
$$

with

$$
G(y, t)=y+\sum t_{j} \vec{n}_{j}
$$

Pulling back by $G$ as in Sect. 2 we get

$$
\begin{aligned}
\left|T_{r}(M)\right| & =\int_{M \times B_{r}(0)}\left(\sum d y_{k} \wedge d \xi_{k}+\sum t_{j} d n_{j}^{\#}+\sum d t_{j} \wedge n_{j}^{\#}\right)^{n} / n ! \\
& =\int_{M \times B_{r}(0)}\left(\sum d y_{k} \wedge d \xi_{k}+\sum t_{j} F_{j}+\sum d t_{j} \wedge n_{j}^{\#}\right)^{n} / n !
\end{aligned}
$$

Although $M \times B(0, r)$ is not strictly speaking a domain in $\mathbb{R}^{n}$, the integral here should be interpreted as an ordinary integral with respect to $(y, t)$ and the Berezin integral 
with respect to $\xi$. We expand the integrand by the trinomial theorem (since all three forms within the parenthesis are of degree 2 they commute), and get

$$
\left|T_{r}(M)\right|=\sum_{l} c_{l, m} \int_{|t|<r} d t \int_{[M]_{s}} \beta^{l} \wedge\left(\sum t_{j} F_{j}\right)^{m-l} .
$$

Here $\beta=\sum d y_{k} \wedge d \xi_{k}$, and we are using

$$
[M] \wedge\left(\sum d t_{j} \wedge n_{j}^{\#}\right)^{n-m}=c[M]_{s} d t_{1} . . \wedge d t_{k}
$$

for some constant $c$. What remains is therefore to compute the form valued integral

$$
\int_{|t|<r}\left(\sum t_{j} F_{j}\right)^{m-l} d t
$$

We first compute the integral

$$
\int_{|t|<r}\left(\sum t_{j} a_{j}\right)^{m-l} d t
$$

where $a=\left(a_{1}, \ldots a_{p}\right)$ lies in $\mathbb{R}^{p}$. It clearly vanishes when $m-l$ is odd, and when $m-l=2 q$ it equals a constant times

$$
|a|^{2 q} r^{2 q+p}
$$

This follows since the integral is rotational invariant and homogenous of degree $2 q$ in $a$, and homogenous of order $2 q+p$ in $r$. Hence, up to a constant, the integral in (10.2) equals

$$
\left(\sum a_{j}^{2}\right)^{q} r^{2 q+p}
$$

From a different point view, we could have expanded the integrand in (10.2) and obtained a linear combination of monomials in $a$ of degree $m-l$. The fact that the resulting homogenous polynomial in $a$ is given by (10.3) is therefore an algebraic identity. This must also hold when $a_{j}$ are not real numbers but lie in any commutative algebra over the reals, like in our case when $a_{j}=F_{j}$ are two-forms. Hence

$$
\int_{|t|<r}\left(\sum t_{j} F_{j}\right)^{m-l} d t=c_{q} r^{2 q+p}\left(\sum F_{j}^{2}\right)^{q}=c_{q}^{\prime} r^{2 q+p} R^{q}
$$

Inserting this into (10.1), the theorem follows.

Acknowledgements Open access funding provided by Chalmers University of Technology. 
Open Access This article is licensed under a Creative Commons Attribution 4.0 International License, which permits use, sharing, adaptation, distribution and reproduction in any medium or format, as long as you give appropriate credit to the original author(s) and the source, provide a link to the Creative Commons licence, and indicate if changes were made. The images or other third party material in this article are included in the article's Creative Commons licence, unless indicated otherwise in a credit line to the material. If material is not included in the article's Creative Commons licence and your intended use is not permitted by statutory regulation or exceeds the permitted use, you will need to obtain permission directly from the copyright holder. To view a copy of this licence, visit http://creativecommons.org/licenses/by/4.0/.

\section{References}

Alexander, H., Osserman, R.: Area bounds for various classes of surfaces. Am. J Math. 97, 753-769 (1975) Babaee, F.: Complex tropical currents, extremality and approximations (2014). arXiv:1403.7456

Bedford, E., Taylor, B.A.: The Dirichlet problem for a complex Monge-Ampère equation. Invent. Math. 37, 1-44 (1976)

Bedford, E., Taylor, B.A.: A new capacity for plurisubharmonic functions. Acta Math. 149, 1-40 (1982)

Berezin, F.: Introduction to superanalysis. Mathematical Physics and Applied Mathematics, vol. 9. D Reidel Publishing Company, Dordrecht (1987)

Berndtsson, B.: Subharmonicity properties of the Bergman kernel and some other functions associated to pseudoconvex domains. Ann. Inst. Fourier 56, 1663-1662 (2006)

Berndtsson, B., Sibony, N.: The $\bar{\partial}$-equation on a positive current. Invent. Math. 147, 371-428 (2002)

Brendle, S., Hung, P.-K.: Area bounds for minimal surfaces that pass through a prescribed point in the ball. Geom. Funct. Anal. 27, 235-239 (2017)

Colding, T.H., Minicozzi II, W.P.: Minimal Surfaces, Courant Lecture Notes in Mathematics, vol. 4 (1999)

Colding, T.H., Minicozzi II, W.P., Pedersen, E.K.: Mean curvature flow. Bull. Am. Math. Soc. 52, 297-333 (2015)

El Mir, H.: Sur le prolongement des courants positifs fermés. Acta Math. 153, 1-45 (1984)

Gray, A.: Tubes. Addison-Wesley, Boston (1990)

Harvey, R., Lawson, B.: Extending minimal varieties. Invent. Math. 28, 209-226 (1975)

Lagerberg, A.: Super currents and tropical geometry. Math. Z. 270, 125-156 (2012)

Landkof, N.: Foundations of Modern Potential Theory. Grundlehren der mathematischen Wissenschaften. Springer, Berlin (1972)

Lelong, P.: Fonctions plurisousharmoniques et formes differentielles positives. Gordon and Breach, New York (1968)

Mikhalkin, G.: Tropical geometery and its applications. Proceedings of the ICM, Madrid (2006)

Skoda, H.: Prolongement des courants, positifs, fermés des masse finie. Invent. Math. 66, 361-376 (1982)

Smoczyk, K.: Mean curvature flow in higher codimension: introduction and survey. Calc. Var. Partial Differ. Equ. 8, 231-274 (1999)

Warner, F.: Foundations of Differentiable Manifolds and Lie Groups. G. T. M. Springer, New York (1983)

Weyl, H.: Zur infinitesmalgeometrie $p$-dimensionale fläche im $n$-dimensionalen Raum. Math. Z. 12, 154 160 (1922)

Weyl, H.: On the volume of tubes. Am. J. Math. 61, 461-472 (1939)

Publisher's Note Springer Nature remains neutral with regard to jurisdictional claims in published maps and institutional affiliations. 